\title{
A GENERALIZATION OF THE CONSERVATION INTEGRAL
}

\author{
VOLKMAR LIEBSCHER \\ Institut für Angewandte Mathematik \\ Fakultät für Mathematik und Informatik \\ Friedrich-Schiller-Universität Jena \\ D-07740 Jena, Germany \\ E-mail: liebscher@minet.uni-jena.de
}

\begin{abstract}
Starting from the scheme given by Hudson and Parthasarathy [7, 11] we extend the conservation integral to the case where the underlying operator does not commute with the time observable. It turns out that there exist two extensions, a left and a right conservation integral. Moreover, Itô's formula demands for a third integral with two integrators. Only the left integral shows similar continuity properties to that derived in [11] used for extending the integral to more than simple integrands.

In another approach we extend the previous notions for the integrals to much larger domains of definition and to much more processes, including anticipating ones. Similar to [5, 10], we use the Skorohod integral and the Malliavin derivative acting on a symmetric Fock space [3, 4]. It appears that this formulation unifies all three integrals in the double integrator one.

1. Introduction. In $[7,11]$ there was developed the well known quantum stochastic calculus. The basic integrators in this theory are the annihilation process $A_{m}$, the creation process $A_{m}^{*}$ and the conservation process $\Lambda_{H}$, where $m$ is a martingale w.r.t. the time observable $\xi$ and $H$ commutes with $\xi$. These properties assure that the basic integrator processes have so called independent increments, at least on a certain exponential domain.

In $[8]$ it turned out that sometimes it should be useful to have conservation integrals even in the case, where $H$ does not commute with $\xi$. The basic observation in that paper was that it is only necessary that the integrand appears at any time to the left of the integrator. Now we want to make a thorough discussion of these integrals. In a first approach we try to make use of the special structure of the integrators related to exponential vectors. Besides the easy going left integral there should also be a right integral adjoint to the former. As we lost the property of independent increments both
\end{abstract}

1991 Mathematics Subject Classification: Primary 81S25; Secondary 60H05, 60H07.

The paper is in final form and no version of it will be published elsewhere. 
integrals are different. It appears that the technique used by [7, 11] and also basic for [2] is not enough to give good estimates for the norm of a simple integral and we cannot extend the integral to others than simple processes. The Itô formula for the left integral demands for a third integral of the kind

$$
\int \vec{\Lambda}_{R^{\prime}}(\mathrm{d} t) X_{t} \overleftarrow{\Lambda}_{R}(\mathrm{~d} t)
$$

i.e. we have an integral which has (formally) two integrators and one integrand between them. Such a form of quantum stochastic integrals was already proposed in [9].

Unfortunately, the double integrator integral shows the same problems as the right integral for the extension to more general processes. Therefore we use another technique to extend the last two integrals to more integrands. This second way is based on the use of the Malliavin derivative (gradient) and Skorohod integral analogous to [5, 10], but we prefer a description almost independent from an underlying $L^{2}$ structure. This way all three integrals appear to be versions of the double integrator integral. Also the Skorohod type number integral of [10] is a special case of such double integrator conservation integrals.

We think this short note may provide some example on the way to a general theory of quantum stochastic integration including also dependent increment processes.

Acknowledgements. Part of the work was done during a visit of the author at the Centro Vito Volterra in Roma. The author wants to thank Luigi Accardi for his kind hospitality and nice discussion on a related subject as well as Michael Skeide for stimulating discussion.

2. Basic definitions and notations. For the natural, real, positive real and complex numbers respectively we use the symbols $\mathbf{N}, \mathbf{R}, \mathbf{R}_{+}=[0, \infty)$ and $\mathbf{C}$ respectively.

The indicator function of a set $B$ is denoted $1_{B}$, the complement of this set by $B^{\mathrm{C}}$.

3. Conservation integrals on the exponential domain. Let us be given a separable Hilbert space $H$ with a so called time observable $\xi$, which is a projection valued measure (in the $\sigma$-strong topology) on $\mathbf{R}_{+}$without atoms. In contrast to the usual assumption we do not suppose that $\xi\left(\mathbf{R}_{+}\right)=\xi_{\mathbf{R}_{+}}=\mathbb{I}$ (I is the identity operator). To simplify notations, we will return to $\xi_{[0, \infty)}=\mathbb{I}$ by setting $\xi_{\{0\}}=\mathbb{I}-\xi_{\mathbf{R}_{+}}$, i.e. $\{0\}$ may be an atom of $\xi$.

Consider the Fock space $\Gamma(H)=\oplus_{n=0}^{\infty} \frac{1}{\sqrt{n !}} H_{\mathrm{sym}}^{\otimes n}$. Here $H_{\mathrm{sym}}^{\otimes n}$ denotes the $n$ fold symmetric tensor product of $H$. The exponential vectors $\mathbf{e}_{u}=\oplus_{n=0}^{\infty} u^{\otimes n}, u \in H$, are of special importance. It is easy to see that $\left\langle\mathbf{e}_{u}, \mathbf{e}_{v}\right\rangle=\mathrm{e}^{\langle u, v\rangle}$. Observe

$$
\Gamma(H) \cong \Gamma\left(H_{B}\right) \otimes \Gamma\left(H_{B^{c}}\right)
$$

for any Borel set $B \subseteq \mathbf{R}_{+}$, where $H_{B}=\xi_{B} H$, coming from $\mathbf{e}_{u} \cong \mathbf{e}_{\xi_{B} u} \otimes \mathbf{e}_{\xi_{B} c} u$. For $u \in H$ we will use the notations $u_{t]}=\xi_{[0, t]} u, u_{(s}=\xi_{(s, \infty)} u$ and $u_{(s, t]}=\xi_{(s, t]} u$.

In the first section we will define our integrals on an exponential domain

$$
\mathfrak{E} \mathfrak{x}(E)=\operatorname{span}\left\{\mathbf{e}_{u}: u \in E \subseteq H\right\},
$$


where the linear space $E$ is $\xi$ invariant: $\xi_{(s, t]} u \in E \forall s, t \in \mathbf{R}_{+}, u \in E$. Moreover, $E$ should be chosen in such a way that $\mathfrak{E} \mathfrak{x}(E)$ is dense in $\Gamma(H)($ e.g. $E=H)$.

Definition 1. Let $\left(X_{t}\right)_{t \in \mathbf{R}_{+}}$be a quantum stochastic process on $\mathfrak{E} \mathfrak{x}(E)$, i.e. $X_{t}$ is a linear operator on $\mathfrak{E x}(E)$ for all $t \in \mathbf{R}_{+}$. We say that $\left(X_{t}\right)_{t \in \mathbf{R}_{+}}$is adapted, if for all $t \in \mathbf{R}_{+}$

$$
X_{t} \mathbf{e}_{u}=X_{t} \mathbf{e}_{u_{t]}} \otimes \mathbf{e}_{u_{(t}} .
$$

The process is called simple if there is an increasing sequence $\left(t_{n}\right)_{n \in \mathbf{N}}$ of real numbers with $t_{0}=0$ and $\lim _{n \rightarrow \infty} t_{n}=\infty$ such that

$$
X_{t}=\sum_{n \in \mathbf{N}} 1_{\left(t_{n}, t_{n+1}\right]}(t) X_{t_{n}}, \quad\left(t \in \mathbf{R}_{+}\right) .
$$

For a contraction $C$ on $H$ we introduce the operator of second quantization $\Gamma(C)$ by

$$
\Gamma(C) \mathbf{e}_{u}=\mathbf{e}_{C u}, \quad(u \in H) .
$$

From [11, section 20] we know that $\left(\Gamma\left(C_{t}\right)\right)_{t \in \mathbf{R}_{+}}$is a strongly continuous semigroup if $\left(C_{t}\right)_{t \in \mathbf{R}_{+}}$is. Denote its generator by $\lambda(S)$ (the conservation operator corresponding to $S)$ if $S$ is the generator of $\left(C_{t}\right)_{t \in \mathbf{R}_{+}}$. As $S \mapsto \lambda(S)$ is linear, we may define $\lambda(S)$ even if $S$ is not sectorial (despite some problem with the domain of definition). The operator $N=\lambda(\mathbb{I})$ is called the number operator. We have $\mathbf{e}_{v} \in \operatorname{dom}(\lambda(S))$ for $v \in \operatorname{dom}(S)$ and

$$
\left\langle\mathbf{e}_{u}, \lambda(S) \mathbf{e}_{v}\right\rangle=\langle u, S v\rangle\left\langle\mathbf{e}_{u}, \mathbf{e}_{v}\right\rangle, \quad(u \in H) .
$$

The annihilation operators $a(f), f \in H$, where

$$
a(f) \mathbf{e}_{u}=\langle f, u\rangle \mathbf{e}_{u}, \quad(u \in H),
$$

also operate on the exponential domain, as well as their adjoints, the creation operators $a^{*}(f)$. For more details cf. [11, Section 20].

We define the left conservation process $\overleftarrow{\Lambda}_{R}$ for $R \in \mathfrak{L}(H)$ by

$$
\overleftarrow{\Lambda}_{R}((s, t])=\lambda\left(\xi_{(s, t]} R\right), \quad\left(s, t \in \mathbf{R}_{+}\right)
$$

Lemma 1. For all $u \in E$

$$
\overleftarrow{\Lambda}_{R}((s, t]) \mathbf{e}_{u}=\mathbf{e}_{u_{s]}} \otimes \psi^{\prime} \otimes \mathbf{e}_{u_{(t}}
$$

where $\Gamma\left(H_{(s, t]}\right) \ni \psi^{\prime}=a^{*}\left(\xi_{(s, t]} R u\right) \boldsymbol{e}_{u_{(s, t]}}$ depends on the whole $u$ and $R$.

Proof. By the properties of exponential vectors we derive

$$
\begin{aligned}
\left\langle\mathbf{e}_{v}, \lambda\left(\xi_{(s, t]} R\right) \mathbf{e}_{u}\right\rangle & =\left\langle v, \xi_{(s, t]} R u\right\rangle\left\langle\mathbf{e}_{v}, \mathbf{e}_{u}\right\rangle \\
& =\left\langle\mathbf{e}_{v_{s]}}, \mathbf{e}_{u_{s]}}\right\rangle\left\langle v_{(s, t]}, \xi_{(s, t]} R u\right\rangle\left\langle\mathbf{e}_{v_{(s, t]}}, \mathbf{e}_{u_{(s, t]}}\right\rangle\left\langle\mathbf{e}_{v_{(t}}, \mathbf{e}_{u_{(t}}\right\rangle
\end{aligned}
$$

This proves the assertion and determines the structure of $\psi^{\prime}$.

Thus $\overleftarrow{\Lambda}_{R}((s, t])$ respects the filtration, but only on the left hand side (after it has acted). 
Definition 2. Let $\left(X_{t}\right)_{t \in \mathbf{R}_{+}}$be a simple adapted process given by (1). We define the left conservation integral $\int_{0}^{t} X_{s} \overleftarrow{\Lambda}_{R}(\mathrm{~d} s)$ as the following operator on $\mathfrak{E} \mathfrak{x}(E)$

$$
\int_{0}^{t} X_{s} \overleftarrow{\Lambda}_{R}(\mathrm{~d} s)=\sum_{n \in \mathbf{N}} X_{t_{n}} \overleftarrow{\Lambda}_{R}\left(\left(t_{n}, t_{n+1} \wedge t\right]\right)
$$

Although $\xi$ may not commute with $R$, we can apply now the same procedure as in [11] to define general integrals. We have only to take care of the fact that $X$ appears in any case to the left of $\lambda\left(\xi_{(s, t]} R\right)$ and the integral has to be taken over $(0, t]$.

LEMma 2. The process $\left(\int_{0}^{t} X_{s} \overleftarrow{\Lambda}_{R}(\mathrm{~d} s)\right)_{t \in \mathbf{R}_{+}}$is a continuous process on $\mathfrak{E} \mathfrak{x}(E)$.

Proof. This follows from the continuity of $t \mapsto \lambda\left(\xi_{(s, t]} R\right) \mathbf{e}_{u}$ and (2).

From the definition it is not clear whether the integral forms itself an adapted process. We see from $(2)$

LEMma 3. The process $\left(\int_{0}^{t} X_{s} \overleftarrow{\Lambda}_{R}(\mathrm{~d} s)\right)_{t \in \mathbf{R}_{+}}$is adapted for all simple adapted processes $\left(X_{t}\right)_{t \in \mathbf{R}_{+}}$if and only if

$$
\xi_{t]} R \xi_{(t}=0, \quad\left(t \in \mathbf{R}_{+}\right) .
$$

Remark 1. There are two simple conditions to fulfil (3). The first one is that $R$ commutes with $\xi$ (clearly, $\left.\xi_{t} \xi_{(t}=0\right)$. This is the condition from the original calculus as laid out in $[7,11]$. There is yet another simple condition, basic in [8], namely $R=R \xi_{\{0\}}$. It appears that selfadjoint operators $R$ allow only for the first possibility.

LEMma 4. If $R=R^{*} \in \mathfrak{L}(H)$ fulfils (3) then $R$ commutes with $\xi$.

Pr o of. By taking the adjoint of $(3)$ we find $\xi_{(t} R \xi_{t]}=0$. Together with (3) this implies

$$
\xi_{t]} R=\xi_{t]} R \xi_{\mathbf{R}}=\xi_{t]} R \xi_{t]}=\xi_{\mathbf{R}} R \xi_{t]}=R \xi_{t]}
$$

for all $t \in \mathbf{R}_{+}$. This is just the assertion.

In the following we will assume (3) as far as we consider the left conservation integral.

If $g, h \in H$ are two vectors,

$$
\langle g, h\rangle(B)=\left\langle g, \xi_{B \backslash\{0\}} h\right\rangle
$$

for all Borel sets $B$ defines an atomless complex valued measure on $\mathbf{R}_{+}$. For simple processes we get the so called first and second fundamental lemmata [11].

Lemma 5. Let $\left(X_{t}\right)_{t \in \mathbf{R}_{+}}$be a simple adapted process. Then

$$
\left\langle\boldsymbol{e}_{u}, \int_{0}^{t} X_{s} \overleftarrow{\Lambda}_{R}(\mathrm{~d} s) \boldsymbol{e}_{v}\right\rangle=\int_{0}^{t}\langle u, R v\rangle(\mathrm{d} s)\left\langle\boldsymbol{e}_{u}, X_{s} \boldsymbol{e}_{v}\right\rangle, \quad(u \in H, v \in E)
$$

Proof. From Lemma 1 we see

$$
\left.\left.\left\langle\mathbf{e}_{u}, X \lambda\left(\xi_{(s, t]} R\right)\right)\right) \mathbf{e}_{v}\right\rangle=\left\langle u_{(s, t]}, \xi_{(s, t]} R v\right\rangle \cdot\left(\left\langle\mathbf{e}_{u_{s]}}, X \mathbf{e}_{v_{s]}}\right\rangle\left\langle\mathbf{e}_{u_{(s, t]}}, \mathbf{e}_{v_{(s, t]}}\right\rangle\left\langle\mathbf{e}_{u_{(t}}, \mathbf{e}_{v_{(t}}\right\rangle\right)
$$

if $X$ is adapted to $\Gamma\left(H_{s]}\right)$. Summation completes the proof. 
Lemma 6. We set $I_{t}^{X}=\int_{0}^{t} X_{s} \overleftarrow{\Lambda}_{R}(\mathrm{~d} s), I_{t}^{Y}=\int_{0}^{t} Y_{s} \overleftarrow{\Lambda}_{R}(\mathrm{~d} s)$ for simple adapted processes $\left(X_{t}\right)_{t \in \mathbf{R}_{+}},\left(Y_{t}\right)_{t \in \mathbf{R}_{+}}$. Then for all $u, v \in E$

$$
\begin{aligned}
\left\langle\int_{0}^{t} X_{s} \overleftarrow{\Lambda}_{R}(\mathrm{~d} s) \boldsymbol{e}_{u}, \int_{0}^{t} Y_{s}\right. & \left.\overleftarrow{\Lambda}_{R}(\mathrm{~d} s) \boldsymbol{e}_{v}\right\rangle=\int_{0}^{t}\langle u, R v\rangle(\mathrm{d} s)\left\langle X_{s} \boldsymbol{e}_{u}, I_{s}^{Y} \boldsymbol{e}_{v}\right\rangle \\
& +\int_{0}^{t}\left\langle\langle R u, v\rangle(\mathrm{d} s)\left\langle I_{s}^{X} \boldsymbol{e}_{u}, Y_{s} \boldsymbol{e}_{v}\right\rangle+\int_{0}^{t}\langle R u, R v\rangle(\mathrm{d} s)\left\langle X_{s} \boldsymbol{e}_{u}, Y_{s} \boldsymbol{e}_{v}\right\rangle\right.
\end{aligned}
$$

Proof. We evaluate $\left.\left.\left.\left.\left\langle X_{1} \lambda\left(\xi_{\left(s_{1}, t_{1}\right]} R\right)\right)\right) \mathbf{e}_{u}, X_{2} \lambda\left(\xi_{\left(s_{2}, t_{2}\right]} R\right)\right)\right) \mathbf{e}_{v}\right\rangle$ if $X_{i}$ is adapted to $\left.\Gamma\left(H_{s_{i}}\right]\right)$ for $i=1,2$. The off-diagonal terms are easily found to coincide with the proposed terms in analogy with the first fundamental lemma. If $s_{1}=s_{2}=s, t_{1}=t_{2}=t$ we have

This gives

$$
\left.\left.X_{i} \lambda\left(\xi_{(s, t]} R\right)\right)\right) \mathbf{e}_{u}=X_{i} \mathbf{e}_{u_{s]}} \otimes \Gamma\left(\xi_{(s, t]}\right) \lambda\left(\xi_{(s, t]} R\right) \mathbf{e}_{u} \otimes \mathbf{e}_{u_{(t}}
$$

$$
\begin{aligned}
& \left.\left.\left.\left.\left\langle X_{1} \lambda\left(\xi_{(s, t]} R\right)\right)\right) \mathbf{e}_{u}, X_{2} \lambda\left(\xi_{(s, t]} R\right)\right)\right) \mathbf{e}_{v}\right\rangle= \\
& \left\langle X_{1} \mathbf{e}_{u}, X_{2} \mathbf{e}_{v}\right\rangle\left(\left\langle\xi_{(s, t]} R u, v\right\rangle\left\langle u, \xi_{(s, t]} R v\right\rangle+\left\langle\xi_{(s, t]} R u, \xi_{(s, t]} R v\right\rangle\right) .
\end{aligned}
$$

A continuity argument like in [11, Proposition 25.2] completes the proof.

Analogously to [11] we call an adapted process stochastically integrable if there is a sequence of simple adapted processes $\left(X_{t}^{n}\right)_{t \in \mathbf{R}_{+}}$on $\mathfrak{E x}(E)$ such that

$$
\lim _{n \rightarrow \infty} \int_{0}^{t}\left\|\left(X_{s}^{n}-X_{s}\right) \mathbf{e}_{u}\right\|^{2}\langle v, v\rangle(\mathrm{d} s)=0, \quad\left(t \in \mathbf{R}_{+}, u \in E, v \in H\right) .
$$

The second fundamental lemma guarantees the existence of the integral for stochastically integrable processes, cf. [11]. Again, we obtain for this $\overleftarrow{\Lambda}_{R}$ integral versions of the Lemmata 5 and 6.

Remark 2. There are two reasons why the technique of [11] for the definition of the $\overleftarrow{\Lambda}$ integral works:

- The integrand appears to the left of the integrator. Thus we can use the (more or less known) action of the conservation operator on exponential vectors.

- $\overleftarrow{\Lambda}_{R}((s, t])$ affects only the ( $s$ part of the exponential vector $\mathbf{e}_{u}$ (see Lemma 1 ). So we can do the calculations directly with $\overleftarrow{\Lambda}_{R}((s, t])$ even if an $\left.s\right]$ adapted operator acts after the integrator term.

Neither of these two conditions is satisfied for the adjoint integrator $\vec{\Lambda}$ (differing from $\overleftarrow{\Lambda})$ considered below. Thus the integrator process $\overleftarrow{\Lambda}$ defined in such a way is not a semimartingale in the sense of [1] or an integrator of scalar type in the sense of [2]. It is only a semimartingale from the left. The adjoint integrator process $\vec{\Lambda}$ will pose severe problems. First, we need an additional condition to define even the simple integral and second, there is no simple second fundamental lemma. 
Remark 3. From the definition it is simple to see that the above defined integral coincides with the conservation integral from [11] in the case where $R$ commutes with $\xi$.

The second fundamental lemma provides some form of a weak Itô formula. We want to search now for the integrals appearing in a strong Itô formula. First we need an adjoint integral to $\overleftarrow{\Lambda}_{R}$. Define the right conservation process $\vec{\Lambda}_{R}$ by

$$
\vec{\Lambda}_{R}((s, t])=\lambda\left(R \xi_{(s, t]}\right), \quad\left(s, t \in \mathbf{R}_{+}\right) .
$$

For the $\overleftarrow{\Lambda}_{R}$ integral the integrand has to appear to the left of the integrator, which comes out to be after adjunction on the right of $\vec{\Lambda}_{R}(\mathrm{~d} s)$. So we would like to define a right conservation integral of a simple process $X$ by

$$
\int_{0}^{t} \vec{\Lambda}_{R}(\mathrm{~d} s) X_{s}=\sum \vec{\Lambda}_{R}\left(\left(t_{n}, t_{n+1} \wedge t\right]\right) X_{t_{n}}
$$

Here the problem appears whether $X_{s} \mathbf{e}_{v} \in \operatorname{dom}\left(\lambda\left(R \xi_{(s, t]}\right)\right)$. First we shall use the closure of the conservation operator. But even then adaptedness is not enough to have this domain relation automatically. We know that $\operatorname{dom}\left(\overline{\lambda\left(R \xi_{(s, t]}\right)}\right) \supseteq \operatorname{dom}(N)=\operatorname{dom}(\lambda(\mathbb{I}))$. So it seems that we have to assume $X_{s} \mathbf{e}_{v} \in \operatorname{dom}(N)$ for all $s \in \mathbf{R}_{+}$. Fortunately, adaptedness helps now to derive something slightly better.

Lemma 7. Suppose $\psi \in \operatorname{dom}\left(\lambda\left(\xi_{s]}\right)^{1 / 2}\right) \cap \Gamma\left(H_{s]}\right)$. Then $\psi \otimes \mathbf{e}_{v_{(s}} \in \operatorname{dom}\left(\overline{\lambda\left(R \xi_{(s, t]}\right)}\right)$ and

$$
\begin{aligned}
\overline{\lambda\left(R \xi_{(s, t]}\right)} \psi \otimes \mathbf{e}_{v_{(s}} & =a^{*}\left(R \xi_{(s, t]} v\right) \psi \otimes \mathbf{e}_{v_{(s}} \\
& =\psi \otimes \overline{\lambda\left(\xi_{(s, t]} R \xi_{(s, t]}\right)} \boldsymbol{e}_{v_{(s}}+a^{*}\left(\left(\mathbb{I}-\xi_{(s, t]}\right) R v_{(s)}\right) \psi \otimes \mathbf{e}_{v_{(s}} .
\end{aligned}
$$

Proof. By looking at matrix elements with exponential vectors we see $\lambda\left(R^{\prime}\right) \mathbf{e}_{v}=$ $a^{*}\left(R^{\prime} v\right) \mathbf{e}_{v}$. Furthermore, $\operatorname{dom}\left(\overline{a^{*}(f)}\right) \supset \operatorname{dom}\left(\lambda(P)^{1 / 2}\right)$ if $P$ is a projector with $P f=f$. This implies that both terms on the right hand side of (5) are well defined and the equation follows from

$$
\lambda\left(R \xi_{(s, t]}\right)=\lambda\left(\xi_{(s, t]} R \xi_{(s, t]}\right)+\lambda\left(\left(\mathbb{I}-\xi_{(s, t]}\right) R \xi_{(s, t]}\right)
$$

on $\mathfrak{E} \mathfrak{x}(E)$ and the fact that $\mathfrak{E} \mathfrak{x}(E)$ is a core of $a^{*}\left(\left(\mathbb{I}-\xi_{(s, t]}\right) R v_{(s)}\right.$.

Definition 3. For a simple adapted process $\left(X_{t}\right)_{t \in \mathbf{R}_{+}}$on $\mathfrak{E x}(E)$ fulfilling

(N) $X_{t} \psi \in \operatorname{dom}(\sqrt{N})$ for all $t \in \mathbf{R}_{+}$and $\psi \in \mathfrak{E x}(E)$

we define the right conservation integral $\int_{0}^{t} \vec{\Lambda}_{R}(\mathrm{~d} s) X_{s}$ by

$$
\int_{0}^{t} \vec{\Lambda}_{R}(\mathrm{~d} s) X_{s}=\sum_{n \in \mathbf{N}} \overline{\lambda\left(R \xi_{\left(t_{n}, t_{n+1} \wedge t\right]}\right)} X_{t_{n}} .
$$

The first fundamental lemma for this integral follows by taking adjoints in Lemma 5 .

LEMMA 8. Let $\left(X_{t}\right)_{t \in \mathbf{R}_{+}}$be a simple adapted process for which $(\mathrm{N})$ is valid. Then

$$
\left\langle\boldsymbol{e}_{u}, \int_{0}^{t} \vec{\Lambda}_{R}(\mathrm{~d} s) X_{s} \boldsymbol{e}_{v}\right\rangle=\int_{0}^{t}\left\langle R^{*} u, v\right\rangle(\mathrm{d} s)\left\langle\boldsymbol{e}_{u}, X_{s} \boldsymbol{e}_{v}\right\rangle, \quad(u \in H, v \in E) .
$$


We want to mention that there is no suitable second fundamental lemma providing us a tool for extending the integral to at least continuous processes. So we have to restrict to simple processes only as far as we want to use the properties of the exponential domain. We will find a more conceptual solution to this problem in Section 4.

LEMma 9. The process $\left(\int_{0}^{t} \vec{\Lambda}_{R}(\mathrm{~d} s) X_{s}\right)_{t \in \mathbf{R}_{+}}$is adapted for all simple adapted processes $\left(X_{t}\right)_{t \in \mathbf{R}_{+}}$if and only if

$$
\xi_{(t} R \xi_{t]}=0 \quad\left(t \in \mathbf{R}_{+}\right)
$$

In the following we will assume (6) to be true as far as right conservation integrals are considered. Again, we get by integration a continuous process.

Lemma 10. The process $t \mapsto \int_{0}^{t} \vec{\Lambda}_{R}(\mathrm{~d} s) X_{s}$ is a continuous process on $\mathfrak{E} \mathfrak{x}(E)$.

Proof. This follows from continuity of the conservation and creation processes as well as from (5).

For simple processes it is immediate that $\vec{\Lambda}_{R^{*}}$ is in the usual sense adjoint to $\overleftarrow{\Lambda}_{R}$ :

LEMMA 11. Let $\left(X_{t}\right)_{t \in \mathbf{R}_{+}}$be a simple adapted process which has an adjoint $\left(X_{t}^{*}\right)_{t \in \mathbf{R}_{+}}$ with $(\mathrm{N})$ on $\mathfrak{E} \mathfrak{x}(E)$ :

$$
\left\langle\psi_{1}, X_{t} \psi_{2}\right\rangle=\left\langle X_{t}^{*} \psi_{1}, \psi_{2}\right\rangle, \quad\left(\psi_{1}, \psi_{2} \in \mathfrak{E} \mathfrak{x}(E)\right)
$$

Then

$$
\left\langle\psi_{1}, \int_{0}^{t} X_{s} \overleftarrow{\Lambda}_{R}(\mathrm{~d} s) \psi_{2}\right\rangle=\left\langle\int_{0}^{t} \vec{\Lambda}_{R^{*}}(\mathrm{~d} s) X_{s}^{*} \psi_{1}, \psi_{2}\right\rangle, \quad\left(\psi_{1}, \psi_{2} \in \mathfrak{E x}(E)\right)
$$

So we can look at the second fundamental lemma for the $\overleftarrow{\Lambda}_{R}$ process as a weak Itô formula connecting the $\vec{\Lambda}_{R^{*}}$ integral and the $\overleftarrow{\Lambda}_{R}$ integral. Thus the first fundamental lemma for a $\vec{\Lambda}_{R^{*}}(\mathrm{~d} s) \overleftarrow{\Lambda}_{R}(\mathrm{~d} s)$ integral should be given by the measure $\langle R u, R v\rangle$, see Lemma 6 . But should the integrator appear left or right? It has to appear (split) on both sides, the $\vec{\Lambda}$ part before, the $\overleftarrow{\Lambda}$ part after the integrand. For simple processes this looks like

$$
\int_{0}^{t} \vec{\Lambda}_{R^{\prime}}(\mathrm{d} s) X_{s} \overleftarrow{\Lambda}_{R}(\mathrm{~d} s)=\lim _{n \rightarrow \infty} \sum_{k \in \mathbf{N}}\left(\vec{\Lambda}_{R^{\prime}}\left(\left(t_{k}^{n}, t_{k+1}^{n} \wedge t\right]\right) X_{t_{k}^{n}} \overleftarrow{\Lambda}_{R}\left(\left(t_{k}^{n}, t_{k+1}^{n} \wedge t\right]\right)\right)
$$

Thereby we take the limit over a sequence of finer and finer partitions.

Lemma 12. Suppose $R, R^{\prime} \in \mathfrak{L}(H)$ are such that $R$ and $R^{\prime *}$ fulfil (3). Further, let $\left(X_{t}\right)_{t \in \mathbf{R}_{+}}$be an adapted simple process on $\mathfrak{E} \mathfrak{x}(E)$ fulfilling $(\mathrm{N})$. Then

$$
\underset{n \rightarrow \infty}{\mathrm{W}-\lim _{k \in \mathbf{N}}} \sum_{k} \lambda\left(R^{\prime} \xi_{\left(t_{k}^{n}, t_{k+1}^{n} \wedge t\right]}\right) X_{t_{k}^{n}} \lambda\left(\xi_{\left(t_{k}^{n}, t_{k+1}^{n} \wedge t\right]} R\right) \mathbf{e}_{v}
$$

exists for all $t>0, v \in E$ (as vector in $\Gamma(H))$.

Proof. Clearly, it is enough to prove this for constant processes. From Lemma 1 we know $X_{s} \overleftarrow{\Lambda}_{R}(s, t) \mathbf{e}_{v}=a^{*}\left(\xi_{(s, t]} R v\right) X_{s} \mathbf{e}_{v}$ for $s<t$. Thus we derive

$$
\vec{\Lambda}_{R^{\prime}}(s, t) X_{s} \overleftarrow{\Lambda}_{R}(s, t) \mathbf{e}_{v}=\lambda\left(R^{\prime} \xi_{(s, t]}\right) a^{*}\left(\xi_{(s, t]} R v\right) X_{s} \mathbf{e}_{v}, \quad\left(v \in E, s<t \in \mathbf{R}_{+}\right)
$$


On a suitable domain the formula $\lambda(S) a^{*}(f)=a^{*}(S f)+a^{*}(f) \lambda(S)$ is valid (cf. [11, Proposition 20.13]). This implies

$$
\lambda\left(R^{\prime} \xi_{(s, t]}\right) a^{*}\left(\xi_{(s, t]} R v\right)=a^{*}\left(R^{\prime} \xi_{(s, t]} R v\right)+a^{*}\left(\xi_{(s, t]} R v\right) \lambda\left(R^{\prime} \xi_{(s, t]}\right)
$$

or applied to $X_{s} \mathbf{e}_{v}$

$$
\vec{\Lambda}_{R^{\prime}}(s, t) X_{s} \overleftarrow{\Lambda}_{R}(s, t) \mathbf{e}_{v}=a^{*}\left(R^{\prime} \xi_{(s, t]} R v\right) X_{s} \mathbf{e}_{v}+a^{*}\left(\xi_{(s, t]} R v\right) \lambda\left(R^{\prime} \xi_{(s, t]}\right) X_{s} \mathbf{e}_{v}
$$

The first summand poses no problems, as the sum over any partition of $(0, t)$ yields $a^{*}\left(R^{\prime} \xi_{(0, t]} R v\right) X_{0} \mathbf{e}_{v}$ (the process $\left(X_{t}\right)_{t \in \mathbf{R}_{+}}$was assumed to be constant). With (5) it follows for the second summand

$$
a^{*}\left(\xi_{(s, t]} R v\right) \lambda\left(R^{\prime} \xi_{(s, t]}\right) X_{s} \mathbf{e}_{v}=a^{*}\left(\xi_{(s, t]} R v\right) a^{*}\left(R^{\prime} \xi_{(s, t]} v\right) X_{s} \mathbf{e}_{v} .
$$

We know

$$
\left\|a^{*}\left(\xi_{(s, t]} R v\right) a^{*}\left(R^{\prime} \xi_{(s, t]} v\right) X_{s} \mathbf{e}_{v}\right\| \leq\left\|\xi_{(s, t]} R v\right\|\left\|R^{\prime} \xi_{(s, t]} v\right\|\left\|\sqrt{\mathbb{I}+N} \sqrt{\mathbb{I}+\xi_{(s}} X_{s} \mathbf{e}_{v}\right\| .
$$

As the adapted process $X$ fulfils (N) we have $\left\|\sqrt{\mathbb{I}+N} \sqrt{\mathbb{I}+\lambda\left(\xi_{(s)}\right.} X_{s} \mathbf{e}_{v}\right\|<\infty$. Therefore

$$
\begin{aligned}
& \left\|\sum_{k \in \mathbf{N}} a^{*}\left(\xi_{\left(t_{k}^{n}, t_{k+1}^{n} \wedge t\right]} R v\right) a^{*}\left(R^{\prime} \xi_{\left(t_{k}^{n}, t_{k+1}^{n} \wedge t\right]} v\right) X_{t_{k}^{n}} \mathbf{e}_{v}\right\| \\
& \leq\left\|\sqrt{\mathbb{I}+N} \sqrt{\mathbb{I}+\xi_{(0}} X_{0} \mathbf{e}_{v}\right\| \sum_{k \in \mathbf{N}}\left\|\xi_{\left(t_{k}^{n}, t_{k+1}^{n} \wedge t\right]} R v\right\|\left\|R^{\prime} \xi_{\left(t_{k}^{n}, t_{k+1}^{n} \wedge t\right]} v\right\| \\
& \leq \frac{1}{2}\left\|\sqrt{\mathbb{I}+N} \sqrt{\mathbb{I}+\xi_{(0}} X_{0} \mathbf{e}_{v}\right\| \sum_{k \in \mathbf{N}}\left(\left\|\xi_{\left(t_{k}^{n}, t_{k+1}^{n} \wedge t\right]} R v\right\|^{2}+\left\|R^{\prime} \xi_{\left(t_{k}^{n}, t_{k+1}^{n} \wedge t\right]} v\right\|^{2}\right) \\
& \leq \frac{1}{2}\left\|\sqrt{\mathbb{I}+N} \sqrt{\mathbb{I}+\xi_{(0}} X_{0} \mathbf{e}_{v}\right\|\left(\left\|\xi_{(0, t]} R v\right\|^{2}+\left\|R^{\prime}\right\|^{2}\left\|\xi_{(0, t]} v\right\|^{2}\right)<\infty .
\end{aligned}
$$

We see that the sums are norm bounded and continuous in the norm $\psi \mapsto\|\sqrt{\mathbb{I}+N} \psi\|$ w.r.t. $X_{0} \mathbf{e}_{v}$. Thus we need only to consider

$$
\lim _{n \rightarrow \infty}\left\langle\mathbf{e}_{u}, \sum_{k \in \mathbf{N}} a^{*}\left(\xi_{\left(t_{k}^{n}, t_{k+1}^{n} \wedge t\right]} R v\right) a^{*}\left(R^{\prime} \xi_{\left(t_{k}^{n}, t_{k+1}^{n} \wedge t\right]} v\right) X_{0} \mathbf{e}_{v}\right\rangle .
$$

It is easy to derive

$$
\begin{aligned}
\left\langle\mathbf{e}_{u}, \sum_{k \in \mathbf{N}} a^{*}\left(\xi_{\left(t_{k}^{n}, t_{k+1}^{n} \wedge t\right]} R v\right) a^{*}\left(R^{\prime} \xi_{\left(t_{k}^{n}, t_{k+1}^{n} \wedge t\right]} v\right) X_{0} \mathbf{e}_{v}\right\rangle & \\
& =\sum_{k \in \mathbf{N}}\left\langle u, \xi_{\left(t_{k}^{n}, t_{k+1}^{n} \wedge t\right]} R v\right\rangle\left\langle\left(R^{\prime}\right)^{*} u, \xi_{\left(t_{k}^{n}, t_{k+1}^{n} \wedge t\right]} v\right\rangle\left\langle\mathbf{e}_{u}, X_{0} \mathbf{e}_{v}\right\rangle
\end{aligned}
$$

and by the argument used in [11, proof of Proposition 25.2] the right hand side tends to zero. We obtain

$$
\underset{n \rightarrow \infty}{\mathrm{w}-\lim _{k \in \mathbf{N}}} \sum_{k} \lambda\left(R^{\prime} \xi_{\left(t_{k}^{n}, t_{k+1}^{n} \wedge t\right]}\right) X_{t_{k}^{n}} \lambda\left(\xi_{\left(t_{k}^{n}, t_{k+1}^{n} \wedge t\right]} R\right) \mathbf{e}_{v}=a^{*}\left(R^{\prime} \xi_{(0, t]} R v\right) X_{0} \mathbf{e}_{v}
$$

completing the proof. 
We may use formula (7) to give

Definition 4. For a simple process $\left(X_{t}\right)_{t \in \mathbf{R}_{+}}$with $(\mathrm{N})$ we define the double integrator conservation integral $\int_{0}^{t} \vec{\Lambda}_{R^{\prime}}(\mathrm{d} s) X_{s} \overleftarrow{\Lambda}_{R}(\mathrm{~d} s)$ on $\mathfrak{E} \mathfrak{r}(E)$ by

$$
\int_{0}^{t} \vec{\Lambda}_{R^{\prime}}(\mathrm{d} s) X_{s} \overleftarrow{\Lambda}_{R}(\mathrm{~d} s) \mathbf{e}_{v}=\sum_{k \in \mathbf{N}} a^{*}\left(R^{\prime} \xi_{\left(t_{k}, t_{k+1} \wedge t\right]} R v\right) X_{t_{k}} \mathbf{e}_{v}, \quad(v \in E) .
$$

LEMma 13. $\left(\int_{0}^{t} \vec{\Lambda}_{R^{\prime}}(\mathrm{d} s) X_{s} \overleftarrow{\Lambda}_{R}(\mathrm{~d} s)\right)_{t \in \mathbf{R}_{+}}$is a continuous process on $\mathfrak{E} \mathfrak{x}(E)$

Lemma 14. Let $\left(X_{t}\right)_{t \in \mathbf{R}_{+}}$be a simple adapted process and $R^{\prime}, R \in \mathfrak{L}(H)$. Then

$$
\left\langle\boldsymbol{e}_{u}, \int_{0}^{t} \vec{\Lambda}_{R^{\prime}}(\mathrm{d} s) X_{s} \overleftarrow{\Lambda}_{R}(\mathrm{~d} s) \boldsymbol{e}_{v}\right\rangle=\int_{0}^{t}\left\langle\boldsymbol{e}_{u}, X_{s} \boldsymbol{e}_{v}\right\rangle\left\langle\left\langle\left(R^{\prime}\right)^{*} u, R v\right\rangle\right\rangle(\mathrm{d} s), \quad(u \in H, v \in E)
$$

Remark 4. We observe by comparing the three first fundamental lemmata that all three conservation integrals come from the double integrator one: $\int_{0}^{t} \vec{\Lambda}_{\mathbb{I}}(\mathrm{d} s) X_{s} \overleftarrow{\Lambda}_{R}(\mathrm{~d} s)=$ $\int_{0}^{t} X_{s} \overleftarrow{\Lambda}_{R}(\mathrm{~d} s)$ and $\int_{0}^{t} \vec{\Lambda}_{R^{\prime}}(\mathrm{d} s) X_{t} \overleftarrow{\Lambda}_{\mathbb{I}}(\mathrm{d} s)=\int_{0}^{t} \vec{\Lambda}_{R^{\prime}}(\mathrm{d} s) X_{s}$. The only drawback is that up to now we have the double integrator integral only for simple processes whereas the left integral exists for all stochastically integrable processes. Section 4 will bridge this gap.

The second fundamental lemma is similarly complicated as that of the $\vec{\Lambda}_{R^{\prime}}$ integral and we will avoid its statement for shortness.

Remark 5. So we face the problem that for two of the main ingredients of a strong Itô formula we can only define matrix elements with exponential vectors and cannot establish the existence as operator. Nevertheless, we can define the integrals using their first fundamental lemmata if this defines reasonable operators. Then under the notations $I_{t}^{X}=\int_{0}^{t} \vec{\Lambda}_{R^{\prime}}(\mathrm{d} s) X_{s}, I_{t}^{Y}=\int_{0}^{t} Y_{s} \overleftarrow{\Lambda}_{R}(\mathrm{~d} s)$ we derive

$$
I_{t}^{X} I_{t}^{Y}=\int_{0}^{t} \vec{\Lambda}_{R^{\prime}}(\mathrm{d} s) X_{s} I_{s}^{Y}+\int_{0}^{t} I_{s}^{X} Y_{s} \overleftarrow{\Lambda}_{R}(\mathrm{~d} s)+\int_{0}^{t} \vec{\Lambda}_{R^{\prime}}(\mathrm{d} s) X_{s} Y_{s} \overleftarrow{\Lambda}_{R}(\mathrm{~d} s)
$$

as soon as all parts of the formula make sense on an exponential domain.

4. Malliavin calculus and conservation integrals. We remind the unbounded operators $\mathcal{S}: H \otimes \Gamma(H) \longmapsto \Gamma(H)$ (Skorohod integral) and $\mathcal{D}: \Gamma(H) \longmapsto H \otimes \Gamma(H)$ (Malliavin derivative). $\mathcal{D}$ is defined on $\operatorname{dom}(\mathcal{D})=\operatorname{dom}(\sqrt{N})$ and extends

$$
\mathcal{D} \mathbf{e}_{h}=h \otimes \mathbf{e}_{h} .
$$

$\mathcal{D}$ defined in this manner is a closed operator, (cf. [3, 4] for a more explicit definition of $\mathcal{D}$ on the whole domain of definition and also [6]). $\mathcal{S}$ is defined as adjoint of $\mathcal{D}$ (there is also an explicit form of $\mathcal{S})$. It is easy to derive that $H \otimes \operatorname{dom}(\sqrt{N}) \subset \operatorname{dom}(\mathcal{S})$ and

$$
\mathcal{S} g \otimes \mathbf{e}_{h}=a^{*}(g) \mathbf{e}_{h} \text {. }
$$

One obtains $\mathcal{S D}=N$ and $\lambda(S)=\mathcal{S} S \otimes \mathbb{I D}$ on $\operatorname{dom}(N)$ for any bounded operator $S$.

Now we want to find a more explicit form of our integrals. Applying the Hahn-Hellinger theorem [11, Section 7] to $\xi$ we may assume $H=H_{\{0\}} \oplus L^{2}\left(\mathbf{R}_{+} \otimes \mathbf{N}, \nu\right)$ where $\nu$ is some 
$\sigma$-finite measure. From a process $\left(X_{t}\right)_{t \in \mathbf{R}_{+}}$we may form the operator $\mathbf{X}$ on

$$
L^{2}\left(\mathbf{R}_{+} \otimes \mathbf{N}, \nu, \Gamma(H)\right) \equiv L^{2}\left(\mathbf{R}_{+} \otimes \mathbf{N}, \nu\right) \otimes \Gamma(H) \subseteq H \otimes \Gamma(H)
$$

by

$$
(\mathbf{X} \psi)(s, k)=X_{s} \psi(s, k)
$$

on the largest domain where this formula makes sense. It is easy to see that $\mathbf{X}$ is densely defined on $\bigcup_{s \in \mathbf{R}_{+}} H_{s]} \otimes \mathfrak{E x}(E)$ for stochastically integrable processes $\left(X_{t}\right)_{t \in \mathbf{R}_{+}}$. We define for $s, t \in \mathbf{R}_{+}$the operator $\mathbf{X}^{(s, t]}=\xi_{(s, t]} \otimes \mathbb{I} \mathbf{X}$ and abbreviate $\mathbf{X}^{(0, t]}$ to $\mathbf{X}^{t]}$.

Proposition 15. (i) Let $R \in \mathfrak{L}(H)$ fulfil (3) and $\left(X_{t}\right)_{t \in \mathbf{R}_{+}}$be a stochastically integrable process on $\mathfrak{E} \mathfrak{x}(E)$. Then on $\mathfrak{E} \mathfrak{x}(E)$

$$
\int_{0}^{t} X_{s} \overleftarrow{\Lambda}_{R}(\mathrm{~d} s)=\mathcal{S} \mathbf{X}^{t]}(R \otimes \mathbb{I}) \mathcal{D}
$$

(ii) Suppose $R \in \mathfrak{L}(H)$ fulfils (6) and $\left(X_{t}\right)_{t \in \mathbf{R}_{+}}$is a simple adapted process on $\mathfrak{E} \mathfrak{x}(E)$ with $(\mathrm{N})$. Then

$$
\int_{0}^{t} \vec{\Lambda}_{R}(\mathrm{~d} s) X_{s}=\mathcal{S}(R \otimes \mathbb{I}) \mathbf{X}^{t]} \mathcal{D} .
$$

(iii) Assume $R, R^{\prime} \in \mathfrak{L}(H)$ fulfil (6) and (3) respectively and the simple adapted process $\left(X_{t}\right)_{t \in \mathbf{R}_{+}}$on $\mathfrak{E} \mathfrak{x}(E)$ obeys $(\mathrm{N})$. Then

$$
\int_{0}^{t} \vec{\Lambda}_{R^{\prime}}(\mathrm{d} s) X_{s} \overleftarrow{\Lambda}_{R}(\mathrm{~d} s)=\mathcal{S}\left(R^{\prime} \otimes \mathbb{I}\right) \mathbf{X}^{t]}(R \otimes \mathbb{I}) \mathcal{D}
$$

Pr o of. Suppose $\left(X_{t}\right)_{t \in \mathbf{R}_{+}}$is a simple process. Then all three left hand sides are well defined by the previous remarks and condition (N) for (ii) and (iii). So we may look only at matrix elements with exponential vectors. Let's have a look at (i).

$$
\begin{aligned}
\left\langle\mathbf{e}_{u}, \mathcal{S} \mathbf{X}(R \otimes \mathbb{I}) \mathcal{D} \mathbf{e}_{v}\right\rangle & =\left\langle\mathcal{D} \mathbf{e}_{u}, \mathbf{X}(R \otimes \mathbb{I}) \mathcal{D} \mathbf{e}_{v}\right\rangle=\left\langle u \otimes \mathbf{e}_{u}, \mathbf{X}(R \otimes \mathbb{I}) v \otimes \mathbf{e}_{v}\right\rangle \\
& =\left\langle u \otimes \mathbf{e}_{u}, \mathbf{X} R v \otimes \mathbf{e}_{v}\right\rangle
\end{aligned}
$$

From $\mathbf{X}=\sum_{n \in \mathbf{N}} \xi\left(\left(t_{n}, t_{n+1}\right]\right) \otimes X_{t_{n}}$ we derive

$$
\begin{aligned}
\left\langle\mathbf{e}_{u}, \mathcal{S} \mathbf{X}^{t]}(R \otimes \mathbb{I}) \mathcal{D} \mathbf{e}_{v}\right\rangle & =\sum_{n \in \mathbf{N}}\left\langle u, \xi\left(\left(t_{n}, t_{n+1} \wedge t\right]\right) R v\right\rangle\left\langle\mathbf{e}_{u}, X_{t_{n}} \mathbf{e}_{v}\right\rangle \\
& =\int_{0}^{t}\langle u, R v\rangle(\mathrm{d} s)\left\langle\mathbf{e}_{u}, X_{s} \mathbf{e}_{v}\right\rangle
\end{aligned}
$$

which is just the proposed value by Lemma 5 . Similarly, we prove (ii) and (iii). If $\left(X_{t}\right)_{t \in \mathbf{R}_{+}}$ is a stochastically integrable process let $\left(X_{t}^{n}\right)_{t \in \mathbf{R}_{+}}$be simple adapted processes converging toward $\left(X_{t}\right)_{t \in \mathbf{R}_{+}}$and denote the respective operators by $\mathbf{X}_{n}$. We know that

and also

$$
\mathbf{X}_{n}^{t]}(R \otimes \mathbb{I}) \mathcal{D} \mathbf{e}_{u} \underset{n \rightarrow \infty}{\longrightarrow} \mathbf{X}^{t]}(R \otimes \mathbb{I}) \mathcal{D} \mathbf{e}_{u}
$$

$$
\mathcal{S} \mathbf{X}_{n}^{t]}(R \otimes \mathbb{I}) \mathcal{D} \mathbf{e}_{u} \underset{n \rightarrow \infty}{\longrightarrow} \int X_{s} \overleftarrow{\Lambda}_{R}(\mathrm{~d} s) \mathbf{e}_{u}
$$

From the closedness of $\mathcal{S}$ we derive the assertion in the general case. 
Definition 5. Assume $\left(X_{t}\right)_{t \in \mathbf{R}_{+}}$is a measurable process on $\operatorname{dom}(X) \subset \Gamma(H)$ and $R, R^{\prime} \in \mathfrak{L}(H)$. Consider the space $\operatorname{dom}\left(\int \vec{\Lambda}_{R^{\prime}}(\mathrm{d} t) X_{t} \overleftarrow{\Lambda}_{R}(\mathrm{~d} t)\right)$ of all $\widehat{\psi} \in \Gamma(H)$ fulfilling

(I1) $\widehat{\psi} \in \operatorname{dom}(\sqrt{N})$.

(I2) It holds $(R \otimes \mathbb{I}) \mathcal{D} \widehat{\psi} \in \operatorname{dom}(\mathbf{X})$.

(I3) $\left(R^{\prime} \otimes \mathbb{I}\right) \mathbf{X}(R \otimes \mathbb{I}) \mathcal{D} \widehat{\psi}$ is in $\operatorname{dom}(\mathcal{S})$.

We define the double integrator conservation integral $\int \vec{\Lambda}_{R^{\prime}}(\mathrm{d} t) X_{t} \overleftarrow{\Lambda}_{R}(\mathrm{~d} t)$ by

$$
\int X_{t} \overleftarrow{\Lambda}_{R}(\mathrm{~d} t) \widehat{\psi}=\mathcal{S}\left(R^{\prime} \otimes \mathbb{I}\right) \mathbf{X}(R \otimes \mathbb{I}) \mathcal{D} \widehat{\psi}, \quad\left(\widehat{\psi} \in \operatorname{dom}\left(\int \vec{\Lambda}_{R^{\prime}}(\mathrm{d} t) X_{t} \overleftarrow{\Lambda}_{R}(\mathrm{~d} t)\right)\right)
$$

Also, we use the notations

$$
\int \vec{\Lambda}_{R}(\mathrm{~d} t) X_{t}=\int \vec{\Lambda}_{R}(\mathrm{~d} t) X_{t} \overleftarrow{\Lambda}_{\mathbb{I}}(\mathrm{d} t) \quad \text { and } \quad \int X_{t} \overleftarrow{\Lambda}_{R}(\mathrm{~d} t)=\int \vec{\Lambda}_{\mathbb{I}}(\mathrm{d} t) X_{t} \overleftarrow{\Lambda}_{R}(\mathrm{~d} t)
$$

Remark 6 . In the same fashion we could define for a measurable field $X: G \ni x \mapsto$ $X_{x}$ integrals like $\int X_{x} \overleftarrow{\Lambda}_{R}(\mathrm{~d} x)$ if $\xi$ is a non-atomic projection valued measure on some (Polish) space $G$ and even multiple integrals in the manner of [10].

Remark 7. Observe that for the definition of the integrals we need neither the assumption of adaptedness of the process $\left(X_{t}\right)_{t \in \mathbf{R}_{+}}$nor the conditions (3) and (6). So we think of the integrals as quantum Skorohod integrals. The choice $R=R^{\prime}=\mathbb{I}$ recovers the (nonadapted) number integral of [10].

Proposition 16. Let $\left(X_{t}\right)_{t \in \mathbf{R}_{+}}$be a measurable process on $\operatorname{dom}(X)$ with adjoint $\left(X_{t}^{*}\right)_{t \in \mathbf{R}_{+}}$. Then for $R, R^{\prime} \in \mathfrak{L}(H)$

$$
\int \vec{\Lambda}_{R^{*}}(\mathrm{~d} t) X_{t}^{*} \overleftarrow{\Lambda}_{R^{\prime *}}(\mathrm{~d} t) \subseteq\left(\int \vec{\Lambda}_{R^{\prime}}(\mathrm{d} t) X_{t} \overleftarrow{\Lambda}_{R}(\mathrm{~d} t)\right)^{*}
$$

Proof. This follows from the adjointness of $\mathcal{S}$ and $\mathcal{D}$ as well as the fact that $\mathbf{X}^{*}$ corresponds to the process $\left(X_{t}^{*}\right)_{t \in \mathbf{R}_{+}}$.

\section{References}

[1] L. Accardi and F. Fagnola, Stochastic integration, in: L. Accardi and W. von Waldenfels (eds.), Quantum Probability and Applications III. Proceedings, Oberwolfach 1987, volume 1303 of Lecture Notes in Mathematics, Springer-Verlag, Berlin Heidelberg New York 1988, pp. 6-19.

[2] L. Accardi, F. Fagnola and J. Quagebeur, A representation free quantum stochastic calculus, J. Funct. Anal. 104 (1992), 149-197.

[3] K.-H. Fichtner and W. Freudenberg, Remarks on stochastic calculus on the Fock space, in: L. Accardi (ed.), Quantum Probability and Related Topics VII, World Scientific Publishing Co., Singapore New Jersey London Hong Kong 1991, pp. 305-323.

[4] K.-H. Fichtner and G. Winkler, Generalized Brownian Motion, Point Processes and Stochastic Calculus for Random Fields, Math. Nachr. 161 (1993), 291-307.

[5] W. Freudenberg, Quantum stochastic integrals on a general Fock space, in: L. Accardi (ed.), Quantum Probability and Related Topics VIII, World Scientific Publishing Co., Singapore 1993, pp. 189-210. 
[6] B. Gaveau and P. Trauber, L'intégrale stochastique comme opérateur de divergence dans l'espace fonctionnel, J. Funct. Anal. 46 (1982), 230-238.

[7] R. L. Hudson and K. R. Parthasarathy, Quantum Itô's formula and stochastic evolution, Commun. Math. Phys. 93 (1984), 301-323.

[8] V. Liebscher, Two Limit Theorems for a Class of Quantum Markov Chains associated to Beam Splittings, submitted to Open Systems and Information Dynamics 1996.

[9] V. Liebscher, On a central limit theorem for monotone noise, submitted to Infinite Dimensional Analysis, Quantum Probability and Related Topics 1997.

[10] J. M. Lindsay, Quantum and Noncausal Stochastic Calculus, Prob. Th. Rel. Fields 97 (1993), 65-80.

[11] K. R. Parthasarathy, An Introduction to Quantum Stochastic Calculus, Birkhäuser, Basel Boston Berlin 1992. 